\title{
V. Sumarios de otras revistas
}




\section{REVISTA DE ADMINISTRACIÓN PÚBLICA}

\section{Sumario del número 166}

(Enero-Abril 2005)

\section{ESTUDIOS}

- T.-R. FERNÁNDEZ: Por una nueva Ley de Expropiación Forzosa y un nuevo sistema de determinación del justiprecio

- M. BULLINGER: El service public francés y la Daseinsvorsorge en Alemania

- A. CASARES MARCOS: La naturaleza fundacional de las cajas de ahorros y la emisión de cuotas participativas

- E. GARCÍA DE ENTERRÍA: Sobre la responsabilidad patrimonial del Estado como autor de una Ley declarada inconstitucional

\section{JURISPRUDENCIA}

\section{COMENTARIOS MONOGRÁFICOS}

- B.-F. MACERA: Examen de algunos casos indemnizatorios no contemplados por la legislación urbanística. Análisis jurisprudencial

- F.L. HERNÁNDEZ GONZÁLEZ: La reserva de ley en materia sancionadora. Avances, retrocesos y contradicciones de la jurisprudencia del Tribunal Constitucional

\section{NOTAS DE JURISPRUDENCIA CONTENCIOSO-ADMINISTRATIVA}

- T. FONT I LLOVET, J. TORNOS MAS y O. MIR PUIGPELAT

\section{CRÓNICA ADMINISTRATIVA}

\section{ESPAÑOLA Y COMUNITARIA}

- F. LÓPEZ RAMÓN: Introducción a los significados de la ordenación del territorio en Europa

- J.E. SORIANO GARCÍA y M.M. SÁNCHEZ GUTIÉRREZ: El control de las ayudas públicas en España: un contexto a cambiar

- S. GONZÁLEZ-VARAS IBÁÑEZ: La gestión territorial

- M. ${ }^{a}$ Y. FERNÁNDEZ GARCÍA: La nueva regulación de la contratación pública en el Derecho comunitario europeo

- I. GONZÁLEZ RÍOS: Sanidad animal. Su vinculación con la ordenación territorial, urbanística y medioambiental

- J. GUILLÉN CARAMÉS: Administración y actividad arbitral: la necesidad de simplificación y reforma de los mecanismos de resolución extrajudicial de conflictos en materia de consumo 


\section{EXTRANJERA}

- S. CIVITARESE MATTEUCCI: El servicio público local en la disciplina del mercado interno de gas natural

- A. CASTRO ESTRADA: Breve crónica del proceso de incorporación de la institución jurídica de la responsabilidad patrimonial del Estado al orden constitucional y legal mexicano

\section{DOCUMENTOS Y DICTÁMENES}

- S. DE LA SIERRA y D. SARMIENTO: La ejecución de las sentencias de los Tribunales contencioso-administrativos

\section{BIBLIOGRAFÍA}

\section{RECENSIONES Y NOTICIAS DE LIBROS}

- ALONSO GARCÍA, R.: El juez español y el Derecho Comunitario. Jurisdicciones constitucional y ordinaria frente a su primacía y eficacia (S. DE LA SIERRA)

- ÁlVAREZ FERNÁNDEZ, M.: El abastecimiento de agua en España (M. ${ }^{a}$ R. ZAMORA ROSELLÓ)

- BOIX PALOP, A.: Las convalidaciones legislativas (J.A. UREÑA SALCEDO)

- CASSAGNE, J.C. (Dir.): Derecho procesal administrativo. Homenaje a Jesús González Pérez (P. GONZÁLEZ SALINAS)

- COLOM PASTOR, B.: Les claus polítiques del procés autonòmic balear (J. MUNAR FULLANA)

- GRAIG, P., y RAWLINGS, R. (eds.): Law and Administration in Europe. Essays in Honour of Carol Harlow (X. ARZOZ SANTISTEBAN)

- DE MIGUEL SÁNCHEZ, N.: Tratamiento de datos personales en el ámbito sanitario: intimidad "versus» interés público (Especial referencia al sida, técnicas de reproducción asistida e información genética) (M. FERNÁNDEZ SALMERÓN)

- MARTÍN DELGADO, I.: El procedimiento por inejecución en la Justicia Europea (I. GALLEGO CÓRCOLES)

- VICENTE Y GUERRERO, G.: El pensamiento político-jurídico de Alejandro Oliván en los inicios del moderantismo en España (C. F. PONCE MARTÍNEZ)

- VV.AA. (Coord. COSCULLUELA MONTANER): Estudios de Derecho Público Económico. Libro Homenaje al profesor Dr. D. Sebastián MARTíN-RETORTILLO BAQUER (M. ${ }^{a}$ I. RIVAS CASTILLO)

- VV.AA. (Coords. JOAN BAUCELLS I LLADÓS y JAUME VERNET I LLO$\mathrm{BET}$ ): La prevención y el control integrados de la contaminación (C. MARTíNRETORTILLO BAQUER)

PRECIOS AÑO 2005

\begin{tabular}{|c|c|c|c|}
\hline \multicolumn{2}{|c|}{ NÚMERO SUELTO } & \multicolumn{2}{c|}{ SUSCRIPCIÓN ANUAL } \\
\hline España & Extranjero & España & Extranjero \\
\hline $18,00 €$ & $25,00 €$ & $51,00 €$ & $74,00 €$ \\
\hline \multicolumn{3}{|c|}{$\begin{array}{r}\text { Números 1-162 (Años 1950-2003), presentados en DVD-ROM: } 250 € \\
\text { y en CD-ROM: } 250 € \\
\text { Suscriptores } 200 €\end{array}$} \\
\hline
\end{tabular}

Suscriptores, venta directa y pedidos por correo de números sueltos: CENTRO DE ESTUDIOS POLÍTICOS Y CONSTITUCIONALES

San Francisco de Sales, 6 28071 MADRID

Tf.: (34) $914412700 \quad$ FAX: (34) 914410086

E-mail: distribrev@cepc.es 


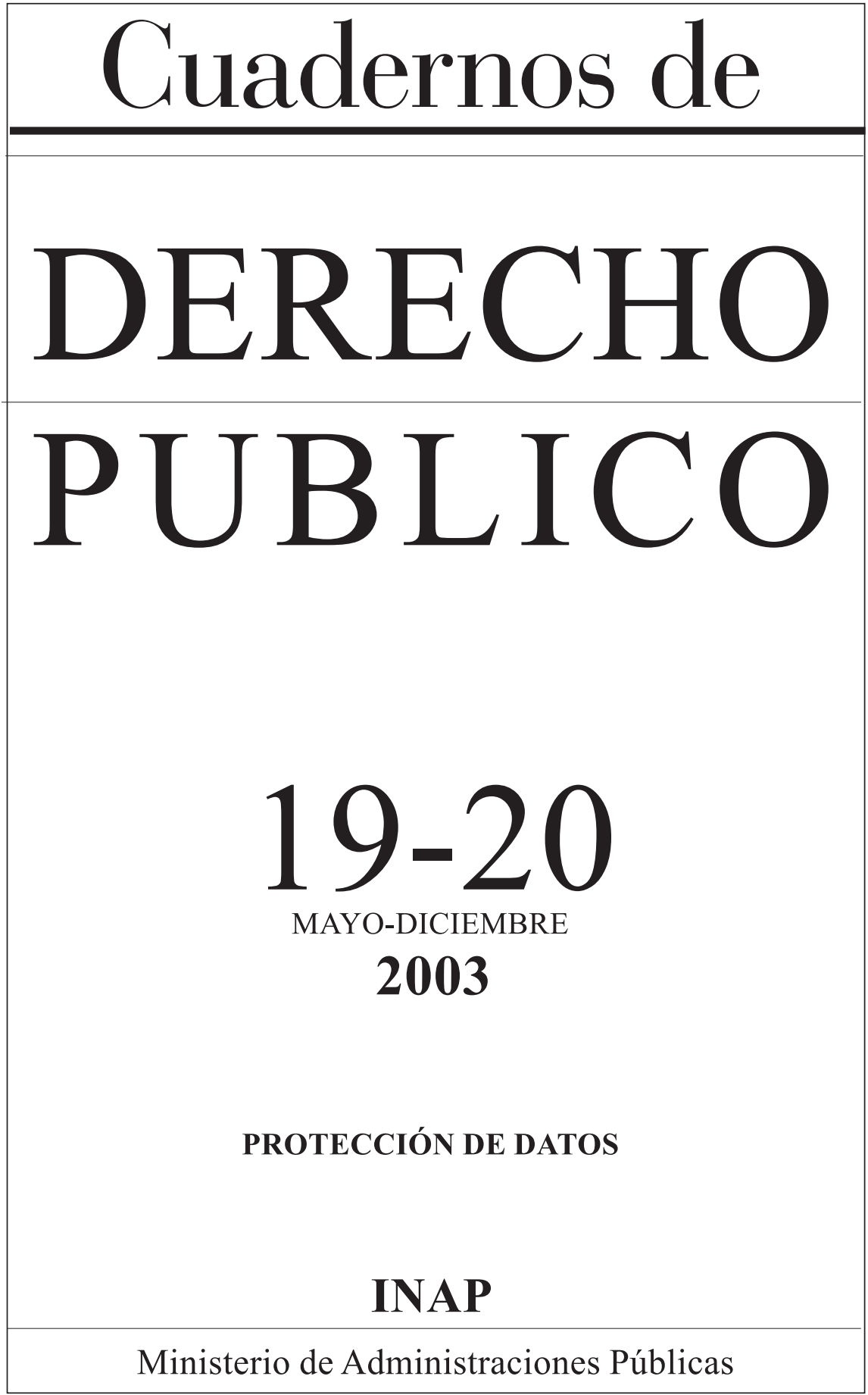




\section{Sumario}

Presentación

\section{Estudios}

Stefano Rodotà: Democracia y protección de datos

Pablo LuCAS MuRILlo De La Cueva: La Constitución y el derecho a la autodeterminación informativa

José Luis PIÑAR MAÑAS: El derecho a la protección de datos de cáracter personal en la jurisprudencia del Tribunal de Justicia de las Cominidades Europeas

José Antonio Seonne Rodríguez: Ética, Derecho y datos personales

Leonardo CERVERA NAVAS: El modelo europeo de protección de datos de carácter personal

Carter MAnNy: La intimidad de Unión Europea y la seguridad de los Estados Unidos: la tensión entre la ley europea de protección de datos y los esfuerzos por parte de Estados Unidos por utilizar los datos sobre pasajeros aéreos para luchar contra el terrorismo y otros delitos

Nelson Remolina ANGaRita: El tratamiento de datos personales para fines estadísticos desde la perspectiva del gobierno electrónico

Eduardo CALvo RoJAs: Algunas consideraciones sobre el procedimiento sancionador en el ámbito de la protección de datos personales

Antonio Troncoso ReIGAda: La protección de datos personales. Una reflexión crítica de la jurisprudencia constitucional

Jesús Rubí NAVARRETE: Tratamiento de datos en el sector de las telecomunicaciones

\section{Documentos}

Fuentes de información sobre protección de datos en Internet, por María José BLANCO ANTÓN 


\title{
REVISTA ARAGONESA DE ADMINISTRACIÓN PÚBLICA
}

\author{
Director: Fernando López Ramón \\ Secretaria: Olga Herráiz Serrano \\ Sumario del número 25 (diciembre 2004)
}

\section{ESTUDIOS:}

- R. DEL ÁGUILA: Tolerancia política y democracia

- A. NOGUEIRA LÓPEZ: La evaluación de la actividad docente e investigadora del profesorado de las universidades públicas

- L.F. BIENDICHO GRACIA: La responsabilidad por daños causados por especies cinegéticas en Aragón

- J.J. SEBASTIÁN LORENTE: El régimen jurídico de los actos y procedimientos administrativos en materia tributaria

\section{DEBATES:}

- J. RODRÍGUEZ-ARANA: Pluralismo y nacionalismo

- J.B. LORENZO DE MEMBIELA: Eficacia, jerarquía y obediencia

- J.M. HERNÁNDEZ DE LA TORRE: Sobre el uso y abuso del término empleados públicos

- S. GORGEMANS Y M. FERNÁNDEZ: Dos décadas de planificación de recursos humanos en la Administración General del Estado

- J. MESEGUER YEBRA: La delegación de firma como técnica de modulación competencial interorgánica

- B. TRÍAS PRATS: La actuación exterior de las Comunidades Autónomas para la cooperación al desarrollo. El marco constitucional

\section{JURISPRUDENCIA:}

- G. GARCÍA-ÁLVAREZ: La valoración del suelo rústico destinado a sistemas generales (a propósito de la Sentencia del TSJ de Castilla y León de 1 de julio de 2004)

- A. BOTO: Sobre la legitimación procesal activa de los colegios profesionales y su titularidad de derechos fundamentales (comentario a la STC de 23 de marzo de 2004)

\section{DERECHO COMPARADO:}

ORGANIZACIÓN TERRITORIAL

- S. RAIMONDI: Evolución y problemas de las regiones en Italia

- S. ESEN: El presente y perspectivas de futuro de la organización territorial del poder político en Turquía

- C. ZOCO ZABALA: Unidad y plurinacionalidad en el modelo territorial del Reino Unido: un estudio comparado con España, Francia e Italia

TURISMO

- D.A. BENÍTEZ: El derecho del Turismo en Argentina

- A. KEMELMAJER: El contrato de turismo en la jurisprudencia reciente de la Unión Europea y Argentina

\section{CRÓNICAS:}

- Crónica de los decimocuartos encuentros del Foro de Derecho Aragonés (I. MURILLO GARCÍA-ATANCE)

\section{BIBLIOGRAFÍA:}

- BREWER-CARÍAS: Ley Orgánica del Tribunal Supremo de Justicia. Procesos y procedimientos constitucionales y contencioso-administrativos, Madrid, 2003 (S. GONZÁLEZVARAS IBÁÑEZ)

- MARTÍN RETORTILLO BAQUER: La interconexión de los ordenamientos jurídicos y el sistema de fuentes del Derecho, Madrid, 2004 (R. SALANOVA ALCALDE)

- PEMÁN GAVÍN: Derecho sanitario aragonés, Zaragoza, 2004 (M.A. BERNAL BLAY)

- VERGARA BLANCO: Derecho eléctrico, Santiago de Chile, 2003 (S. GONZÁLEZ-VARAS IBÁÑEZ)

Precio de la suscripción anual: 21,04 euros + IVA

Número suelto: 12,02 euros + IVA

Suscripciones: $\quad$ REVISTA ARAGONESA DE ADMINISTRACIÓN PÚBLICA INSTITUTO ARAGONÉS DE ADMINISTRACIÓN PÚBLICA Diputación General de Aragón

Paseo María Agustín, 36, 50004 Zaragoza 


\section{ADMINISTRACIÓN DE ANDALUCÍA Revista Andaluza de Administración Pública \\ N. ${ }^{\circ} 54$ \\ Abril-Mayo-Junio 2004 \\ ESTUDIOS \\ Javier García Fernández La Ley del Patrimonio Histórico de 1985: Propuestas para una posible reforma \\ Ínigo del Guayo Castiella Suelo urbano, planeamiento urbanístico y derecho transitorio en la Ley de Ordenación Urbanística de Andalucía \\ Manuel J. Sarmiento Acosta La insularización de la gestión del agua en Canarias: Los Consejos Insulares de Aguas \\ Sebastián Olmedo Pérez Las transferencias de aprovechamientos urbanísticos}

\section{JURISPRUDENCIA}

\section{Comentarios de Jurisprudencia}

La recepción por el Tribunal Supremo de la Doctrina de la Vinculación Negativa de la Ordenanza Local al Principio de Legalidad Sancionadora Francisco Toscano Gil

\section{Notas de Jusrisprudencia \\ Tribunal Constitucional}

Francisco Escribano López

I. Constitución. II. Derechos y Libertades. III. Principios Jurídico Básicos. IV. Instituciones del Estado. V. Fuentes. VI. Organización Territorial del Estado. VIII Economía y Hacienda.

Tribunal Supremo y Tribunal Superior de Justicia de Andalucía

José I. López González (Tribunal Supremo) José L. Rivero Ysern (Tribunal Superior de Justicia de Andalucía) I. Acto Administrativo. II. Administración Local. III. AdministraciónPública. IV Bienes Públicos. V. Comunidades Autónomas. VI. Contratos. VII. Corporaciones de Derecho Público. VIII. Cultura, Educación, Patrimonio Histórico. IX. Derecho Administrativo Económico. X. Derecho Administrativo Sancionador. XI. Derechos Fundamentales y Libertades. XII. Expropiación Forzosa. XIII. Fuentes. XIV. Hacienda Pública. XV. Jurisdicción ContenciosoAdministrativa. XVI. Medio Ambiente. XVII. Organización. XVIII. Personal. XIX. Procedimiento Administrativo. XX: Responsabilidad. XXI. Salud y Servicios Sociales. XXI. Urbanismo y Vivienda. 


\section{CRÓNICA PARLAMENTARIA}

Manuel Medina Guerrero

\section{DOCUMENTOS}

Jaime Rodríguez Arana La Administración Pública y Naciones Unidas

Andrés Navarro Galera Un nuevo modelo para la valoración de las inversiones de las Administraciones Públicas

Rosario Cabañete Pozo Las Cajas de Ahorros Andaluzas: Situación actual y perspectivas futuras

M. ${ }^{a}$ del Mar Caraza Cristín

El aseguramiento de la responsabilidad patrimonial de las Administraciones Públicas: ¿un tema completamente resuelto tras la reforma de la Ley Orgánica 19/2003?

\section{RESEÑA LEGISLATIVA}

Lucía Millán Moro, Antonio Jiménez Blanco, Juan Antonio Carrillo Donaire Disposiciones de las Comunidades Europeas.

Disposiciones Estatales. Disposiciones generales de la Junta de Andalucía Índice Analítico

\section{NOTICIAS DE LA ADMINISTRACIÓN AUTONÓMICA}

Informes: La Agencia Andaluza de la Energía ¿el retorno ficticio del Derecho administrativo?

José I. Morillo-Velarde Pérez, Carmen Núñez Lozano Otras disposiciones de interés para la Administración Autonómica

\section{NOTICIA DE REVISTAS AUTONÓMICAS}

Jesús Jordano Fraga

\section{BIBLIOGRAFÍA}

\section{RECENSIONES}

AA.VV: Estudios sobre ley de Prevencion de Control Integrados de la contaminación. Ed. Aranzadi, Pamplona 2003, 242 págs. 


\section{REVISTA VALENCIANA D'ESTUDIS AUTONÒMICS}

\section{N.우 38 - PRIMER TRIMESTRE DE 2002}

Director: Ramón Martín Mateo

Coordinador General: Rafael Blasco Castany

Secretario de Redacción: Vicente Pérez Plaza

\section{PRESENTACIÓN}

EI Sistema Valenciano de Innovación en el Informe Anual 2002 del Alto Consejo Consultivo en $\mathbf{I}+\mathrm{D}$ de la Presidencia de Generalitat Valenciana

por Manuel López Estornell

- Análisis de las actividades de Investigación y Desarrollo y de Cooperación entre las comunidades académica y empresarial de la Comunidad Valenciana

por Adela García Aracil, Ignacio Fernández de Lucio, Joaquín Ma Azagra Caro, Manuel López Estornell

- La ciencia y tecnología de los materiales en la Comunidad Valenciana. Un análisis de los grupos de investigación por Antonio Gutiérrez Gracia, Elena Castro Martinez, Fernando Jiménez Sáez, Olga Minguez López

— El sector empresarial valenciano asociado al área de materiales por Antonio Gutiérrez Gracia, Carlos Dema Pérez, Fernando Jiménez Sáez, M. ${ }^{a}$ Ángeles Bernabeu Valdés

- La producción científico-técnica de la Comunidad Valenciana por Julia Osca Lluch, Elena Castro Martínez, Ignacio Fernández de Lucio, Pedro Serra Sister

\section{OTRAS SECCIONES}

Colaboran en este número

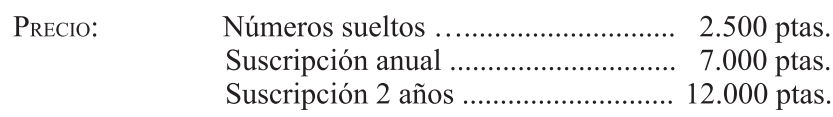

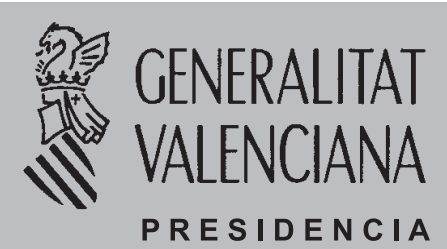

